\title{
Sorting Activities in Plant Cells
}

\author{
Felix Kessler* and J.-M. Neuhaus*
}

\begin{abstract}
Eucaryotic cells (plants, animals, fungi, etc.) are subdivided in membrane-bound compartments (organelles), such as the nucleus, mitochondria, chloroplasts, vacuoles, etc. Most organellar proteins are encoded in the nucleus and synthesized in the cytoplasm. Proper sorting of proteins is required to establish and maintain organellar identity. Molecular machineries at the organelle surfaces specifically recognize targeting sequences of their cognate proteins and mediate their translocation across membranes. Proteins destined for the vacuoles are first translocated across the endoplasmic reticulum membrane, packaged into vesicles, transported to the Golgi, where they are sorted into specific vesicles and subsequently carried to the different types of vacuoles. Though plant cells share many features with animal and yeast cells, chloroplasts and distinct lytic and storage vacuoles are unique to plants. Here, we discuss import of proteins into the chloroplast as well as selective sorting of proteins to either the lytic or the storage vacuole.
\end{abstract}

Keywords: Chloroplast $\cdot$ Mutants $\cdot$ Reporter protein $\cdot$ Targeting $\cdot$ Vacuoles

\section{Chloroplast Protein Import}

The chloroplast, site of photosynthesis, is the best known member of a family of organelles termed plastids. Light induces the rapid differentiation of chloroplasts from dark-grown etioplasts, non-photosynthetic precursor plastids in leaf mesophyll cells. In particular, extensive thylakoid membranes harboring the photosystems are formed. While chloroplasts, generally accepted to be derived from endosymbiotic cyanobacteria, retain around 100 genes in the 'plastome', most of its 2000 or so protein constituents are encoded in the nucleus and synthesized as precursors in the cytosol. The majority of precursor proteins are equipped with $\mathrm{N}$-terminal targeting sequences termed transit peptides. Upon import, transit peptides are cleaved at a specific site by the stromal processing

\footnotetext{
${ }^{*}$ Correspondence: Prof. F. Kesslera, Prof. J.-M. Neuhaus ${ }^{\mathrm{b}}$

aLaboratoire de Physiologie Végétale Tel.: +4132718 2292 Fax: +41327 182271

E-Mail: felix.kessler@unine.ch

bLaboratoire de Biochimie

Institut de Botanique

Université de Neuchâte

Rue Emile-Argand 11

$\mathrm{CH}-2007$ Neuchâtel

Tel.: +41327182207

Fax: +41327 182201

E-Mail: jean-marc.neuhaus@unine.ch
}

peptidase, SPP, resulting in the mature chloroplast protein (Fig. 1). Proteins may then be targeted to the thylakoid membranes or lumen by secondary targeting signals becoming exposed by the cleavage of the transit peptide. Interestingly, the targeting pathways to the thylakoids, Sec, SRP, and Tat, are conserved from bacteria lending further support to the endosymbiont origin of the chloroplast [1][2].

Transport of proteins across the envelope membranes of the chloroplast is energized by ATP and GTP and facilitated by the translocons at the outer membrane of the chloroplast (Toc-complex) and at the inner membrane (Tic-complex) (Fig. 1). In recent years, the molecular nature of a number of components of the Toc- and Tic-complexes has been determined and their physiological role in chloroplast biogenesis elucidated. While all of the principal components were identified using isolated pea chloroplasts as the experimental system, some recent studies used Arabidopsis thaliana as model system, taking advantage of powerful molecular genetic tools, such as gene disruption [3][4].

\section{The Tic-complex}

A number of components of the Ticcomplex have been identified, Tic110, Tic62, Tic55, Tic22 and Tic20 [4][5] (Fig. 1). The exact roles of these proteins, however, are poorly understood or, as in the case of Tic110, controversial. Tic110 was first identified through its association with a late translocation intermediate, a precursor protein arrested while spanning both the outer and inner envelope membranes (Fig. 1) [6]. Its association with a late translocation intermediate, but not with an early intermediate, spanning only the outer membrane, suggests that Tic110 functions in the final stages of chloroplast protein import. Although the topology of Tic110 is a matter of debate, the majority of evidence points to two neighboring, $\mathrm{N}$-terminal transmembrane helices with the C-terminal bulk of Tic110 facing the stroma [7][8]. Association of Tic 110 with ClpC and cpn60 (Fig. 1), suggests that Tic110 may recruit chaperones to the import site to assist in folding of newly imported precursor proteins [7][9]. Reconstitution of recombinant Tic110 into lipid bilayers followed by electrophysiological measurements, however, suggests that Tic110 may function as a protein conducting channel [10]. The proposed channel would require the C-terminal portions of Tic110, considered to be soluble, to insert into the inner membrane possibly in a $\beta$-barrel conformation. Thus, both function and topology of Tic110 require further investigation.

Tic55 [11] and Tic62 [12] (Fig. 1) have been identified as stromal Tic110 interacting proteins. The presence of redox-motifs in these two proteins suggest a potential function in redox-regulation of chloroplast protein import. 


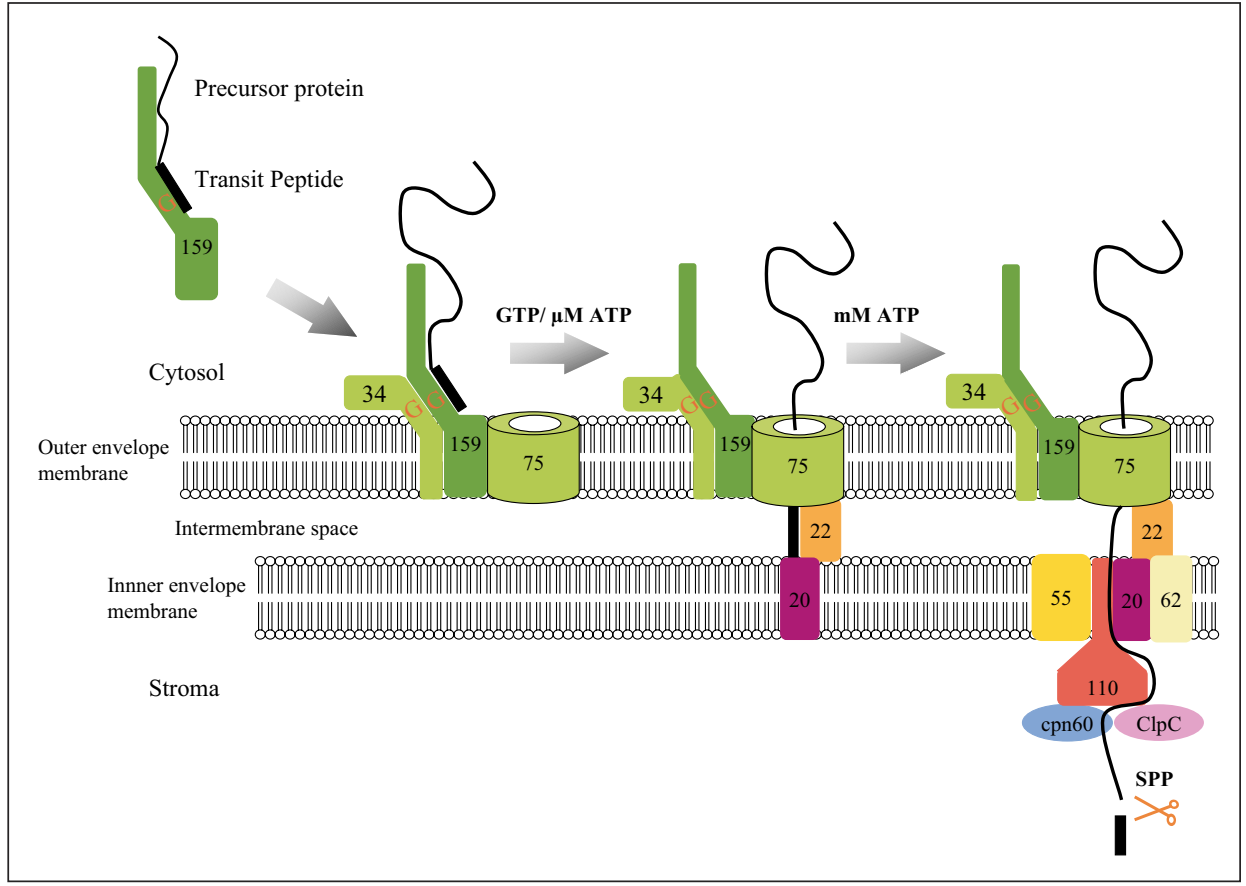

Fig. 1. Functional model of the chloroplast protein import machinery. Import occurs in three stages. First, the transit peptide of a precursor binds to Toc159 in an energy independent fashion. The existence of a cytosolic form of Toc159 suggests that it may also function as a cytosolic precursor receptor. Second, the precursor inserts across the Toc75 channel at the outer membrane in the presence of GTP and low concentrations of ATP $(50 \mu \mathrm{M})$ and makes contact with components at the inner membrane Tic22 and Tic20. In the third stage, requiring high concentrations of ATP $(1 \mathrm{mM})$ in the chloroplast stroma, the precursor translocates across both membranes simultaneously. Tic 110 and associated components, such as Tic55 and Tic62, are recruited to the import machinery. Chaperones (cpn60, ClpC), recruited to the import machinery by Tic110, may assist in folding newly imported proteins or help to energize the import process. Alternatively, Tic110 has been suggested to function as a protein conducting channel at the inner membrane. Inside the chloroplast, stromal processing peptidase (SPP) cleaves transit peptides, resulting in mature chloroplast proteins.

Tic22 and Tic20 were identified by chemical crosslinking to a precursor at the early intermediate stage before traversing the inner membrane [13]. Indeed, Tic22 is located at the inner membrane surface, facing the inter membrane space, supporting an inner membrane precursor receptor function. Tic20 may potentially function as part of a protein conducting channel as it is largely buried in the inner membrane.

\section{The Toc-complex}

The Toc-complex in pea is widely considered to be trimeric, consisting of two homologous GTP-binding proteins, Toc159 and Toc34, and a protein conducting channel component, Toc75 [6] (Fig. 1). Toc159 and Toc34 are exposed at the chloroplast surface, controlling access to Toc75, the channel at the outer membrane. It is probable that the function of Toc159 and Toc34 explains the GTP-requirement of protein import. Indeed, in vitro reconstitution of the three Toc proteins suggests that they are sufficient for translocation of precursors across a lipid bilayer [14]. Direct interactions between the precursor and the components of the Toc-complex during the course of import, suggest a sequence of events during import. At the earliest stages of import, not requiring energy in the form of either GTP or ATP, the transit sequence of the precursor binds to Toc159 [15]. In the presence of GTP, the precursor inserts across the outer membrane making contacts with Toc75, but also with Toc159, involving both the transit peptide and mature portions of the protein. Though recombinant Toc34 binds to precursor proteins in vitro [16], only a single study reports a direct interaction during import into isolated chloroplasts, but with the mature part of the precursor [13]. In summary, most of the available data supports a model in which Toc159 functions as a primary receptor, passing on and inserting precursors into the Toc75 channel, probably via a transient interaction of precursors with Toc34 (Fig. 1).

The crystal structure of Toc34 [17] and biochemical experimentation in Arabidopsis have shed light on the molecular interaction between Toc34 and Toc159 (as well as the corresponding Arabidopsis orthologs, atToc33 and atToc 159 , respectively) [18][19]. The crystal structure revealed that Toc34 forms GDP-bound homodimers.
Amino acid residues interacting with the GDP molecule bound to the other monomer suggest that dimerization may be regulated by GTP-binding and -hydrolysis. Furthermore, homodimerization of Toc34 involves a dimerization motif (D1) conserved in Toc159 as well as in the Arabidopsis orthologs of the two proteins but not present in other GTPases. While the role of Toc34 homodimers remains unclear, the D1 motifs in Toc159 and Toc34 may also account for heterodimerization, including the ability of the isolated, recombinant G-domains of the Arabidopsis proteins to interact [20][21]. The crystal structure of Toc34 suggests that an arginine-residue (R128) close to the D1 motif may function in mutual GTPase activation upon dimerization [17], but this has not been substantiated experimentally. GTPase activity of Toc34 may not only be affected by dimerization but also by a cycle of phosphorylation and dephosphorylation as well as precursor binding [16]: GTPase activity of Toc34 is inactivated by phosphorylation and reactivated by dephosphorylation and strongly stimulated by the presence of precursor proteins. In summary, the structural and biochemical data suggest that regulated GTPase activities control transfer of precursor proteins from the GTPases, Toc159 and Toc34, to the channel Toc75.

\section{Soluble Toc159}

In contrast to Toc 75 and Toc34, Toc 159 also exists in a soluble, cytosolic form [20]. The function of soluble Toc159 is not well understood and it may function either as a cytosolic precursor receptor (Fig. 1) or a soluble pool of the protein ready for assembly into the Toc-complex. Association of cytosolic Toc159 with the Toc-complex requires GTP-binding and -hydrolysis and involves Toc34 as a receptor at the chloroplast surface [18][19].

\section{Knock-out of atToc33 and atToc159}

Gene disruption in Arabidopsis has shed light on the physiological roles of atToc33 and atToc159, the Arabidopsis orthologs of Toc34 and Toc159, respectively. The mutants have been termed ppil (plastid protein import mutant 1, lacking atToc33) [22] and ppi2 (lacking atToc159) [23]. Both mutants are affected in chloroplast biogenesis. ppil has a pale green phenotype, indicative of delayed chloroplast biogenesis. ppi2 is an albino mutant unable to develop photosynthetic plastids. Despite strongly reduced accumulation of photosynthetic proteins, housekeeping proteins appear to be imported normally into ppi 2 plastids. This finding suggests substrate specificity of import receptors as well as the activity of additional 
protein import pathways that may involve the three homologs of atToc159 (atToc132, -120 and -90) [3][23].

A current study, using spontaneous, transgene-induced silencing (occuring in only part of the transgenic population) to suppress the function of Toc159, suggests that Toc159 is not only required for chloroplast biogenesis, but also for chloroplast maintenance. The function of Toc159 may therefore be required for continuous import of photosynthetic proteins into existing chloroplasts (F. Kessler, unpublished results, Fig. 2)

\section{Outlook}

In recent years, likely most of the principal components of the chloroplast protein import machinery have been identified. Moreover, the role of a number of these components in chloroplast biogenesis has been elucidated. However, further experimentation is required to clarify the exact roles of Tic110 and Toc159 in chloroplast protein import. Although much progress has been made in understanding the GTPase cycles of both Toc159 and Toc34 (atToc159 and atToc33), the exact role of GTPase homo- and heterodimerization in import remains open. Another question requiring attention is the role of the homologs of atToc159 in import. Are these proteins substrate specific import receptors and if, how do they differentiate between different classes of import substrates, such as the photosynthetic and housekeeping proteins?

\section{The Plant Secretory System and the Vacuoles}

Eucaryotic cells are equipped with a complex system of membrane-bound organelles that are related and inter-connected by a transport system of vesicles that can bud from one compartment and fuse to another. The mother compartment of the secretory system is the endoplasmic reticulum, at the membrane of which synthesis and import of proteins occurs. A stream of vesicles then selectively carries proteins to the Golgi apparatus, the major sorting center of the system. During their passage through the Golgi, proteins are often modified and sorted to different exit vesicles that will carry them to their different target organelles. For most of these anterograde transport steps, there is a corresponding retrograde transport system for proteins that may have been missorted or need to be recycled, such as the sorting receptors that confer selectivity to specific anterograde transport steps.

The target organelles of the secretory pathway include the plasma membrane, the nuclear envelope, lysosomes (in animal cells), vacuoles (in fungi and plants). Secretion out of the cell of soluble compounds and proteins as well as many components of an extracellular matrix or a cell wall and endocytosis are also functions of the secretory pathway.

\section{The Vacuoles}

Plant cells are often much larger than animal cells. The major reason for this dif-

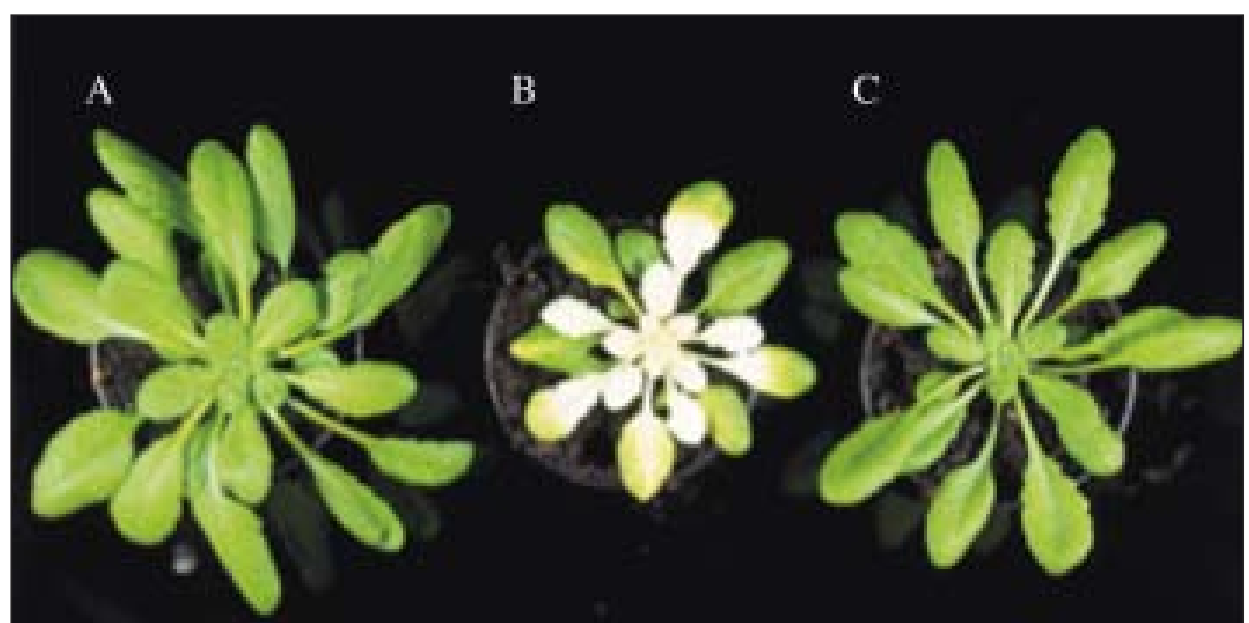

Fig. 2. Toc159 is required for chloroplast maintenance. A. 3 week-old wild type Arabidopsis plant. B. Transgenic Arabidopsis plant engineered to express a truncated version of atToc159 ('Toc86'), exhibiting a silencing phenotype. C. Transgenic Arabidopsis plant engineered to express a truncated version of atToc159 ('Toc86'), exhibiting a wild type phenotype. Note that the plant shown in B originally had the wild type phenotype and normal chloroplasts. Occasional, spontaneous silencing of the endogenous atToc159 gene results in an albino phenotype caused by chloroplast deficiency (note that the transgene-induced defect is only observed in a fraction of the population). The result suggests that continuous import of photosynthetic proteins mediated by Toc159 is essential for chloroplast maintenance. ference is the presence of one or several vacuoles, which may take up more than $95 \%$ of the volume of the cell [24]. Vacuoles lack internal structure, appearing 'empty' under the light or electron microscope (hence their name). In fact, the vacuolar liquid contains salts, sugars, as well as proteins and other substances. These solutes are responsible for the osmotic pressure (turgor) that confers rigidity to the non-woody tissues of plants. In this respect, vacuoles function much like the inner tube of a bicycle tire. Fruit juices are mostly vacuolar sap.

Plant vacuoles have a number of functions that do not always appear compatible: storage (polysaccharides, proteins, pigments), waste disposal (sequestration of toxic compounds), digestion (by proteases, nucleases, glycosidases, lipases) and protection (defense compounds and enzymes). Digestion is the main function of the related lysosomes in animal cells. Incompatible functions may be segregated and carried out in the vacuoles of different cell types, or in different vacuole types within the same cell. The simultaneous presence of two different vacuoles in a single cell was known for some specialized cells such as the pulvini of Mimosa pudica, responsible for the fast movement of their leaflets. Recent experiments indicated that occurrence of different vacuole types in a single cell is far more common than previously thought [25].

\section{Protein Targeting to Vacuoles}

Like most soluble secretory proteins, vacuolar proteins are synthesized with an $\mathrm{N}$-terminal signal sequence that causes the ribosome to bind to the membrane of the endoplasmic reticulum and to push the nascent proteins into its lumen (Fig. 3). Analysis of precursors for several vacuolar proteins and fusion experiments with reporter proteins revealed the existence of additional, specific vacuolar sorting signals, generally included in propeptides (i.e. sequences absent from the mature proteins). Mutagenesis and pharmacological experiments indicated the existence of at least two classes of sorting signals: a sequence-specific signal containing an essential isoleucine (or leucine) and a $\mathrm{C}$-terminal signal requiring accessibility from the polypeptide's end. A third sorting system may rely on protein aggregation, as known in secretory granules of animal cells [26]. However, until recent$1 y$, the specific functions of the sequencespecific signal and the C-terminal signal were not known. Fusion of either type of signal to the reporter protein GFP (green fluorescent protein, originating from a jelly fish) causes the appearance of fluorescent vacuoles in some plants cells [27]. In trans- 
genic Arabidopsis thaliana [28], the sequence-specific signal addresses GFP to the large central vacuole in epidermal cells, but not in guard cells, the cells controlling the gas exchange of leaves, nor in the mesophyll cells which perform most of the photosynthesis (Fig. 4 A). In contrast, the C-terminal sorting signal addresses GFP to the central vacuoles of guard cells and mesophyll cells, while in epidermal cells it accumulates in small peripheral vacuoles (Fig. 4 B).

\section{Protein Sorting to Lytic, Acidic Vacuoles}

The sequence-specific vacuolar sorting system to lytic, acidic vacuoles resembles the sorting system to lysosomes in animal cells and to vacuoles in yeast. Sorting is mediated by a small family of vacuolar sorting receptors (VSRs) [29] with a domain organization similar to animal (mannose-6-phosphate receptors) and yeast (Vps10p) receptors, but lacking homology in their primary sequences. The N-terminal luminal part of these receptors binds to a sorting signal-containing protein in a $\mathrm{pH}$ dependent manner. It is connected by a single transmembrane segment to a $\mathrm{C}$-terminal domain, exposed at the cytosolic face of the membrane and containing a tyrosine-based motif recognized by proteins involved in the formation of clathrin-coated vesicles. The plant receptor BP-80 was indeed first isolated from clathrin-coated vesicles of pea cotyledons based on its $\mathrm{pH}$-dependent substrate binding. The receptor was detected in the Golgi as well as in a prevacuolar (or endosomal) compartment. The lower $\mathrm{pH}$ of this compartment presumably dissociates the ligand from the receptor, which then shuttles back to the Golgi compartment. Arabidopsis thaliana has a small gene family of seven VSRs, which may have different binding specificities and are expressed differentially in different tissues [30].

\section{Protein Sorting to Neutral Vacuoles}

In the case of neutral vacuoles, the corresponding C-terminal sorting signal is recognized by a different system, for which a putative receptor (another small gene family) was recently identified. The neutral vacuole seems to have a $\mathrm{pH}$ closer to that of the Golgi, so acidification is probably not required to dissociate the ligand from its receptor. The type of transport vesicle is not yet known, nor has the analysis of the putative receptor identified so far any motifs (such as a tyrosine-based motif, see above) related to vesicle formation and recruitment of a coat. The relationship to the smooth (non-coated) dense vesicles observed in

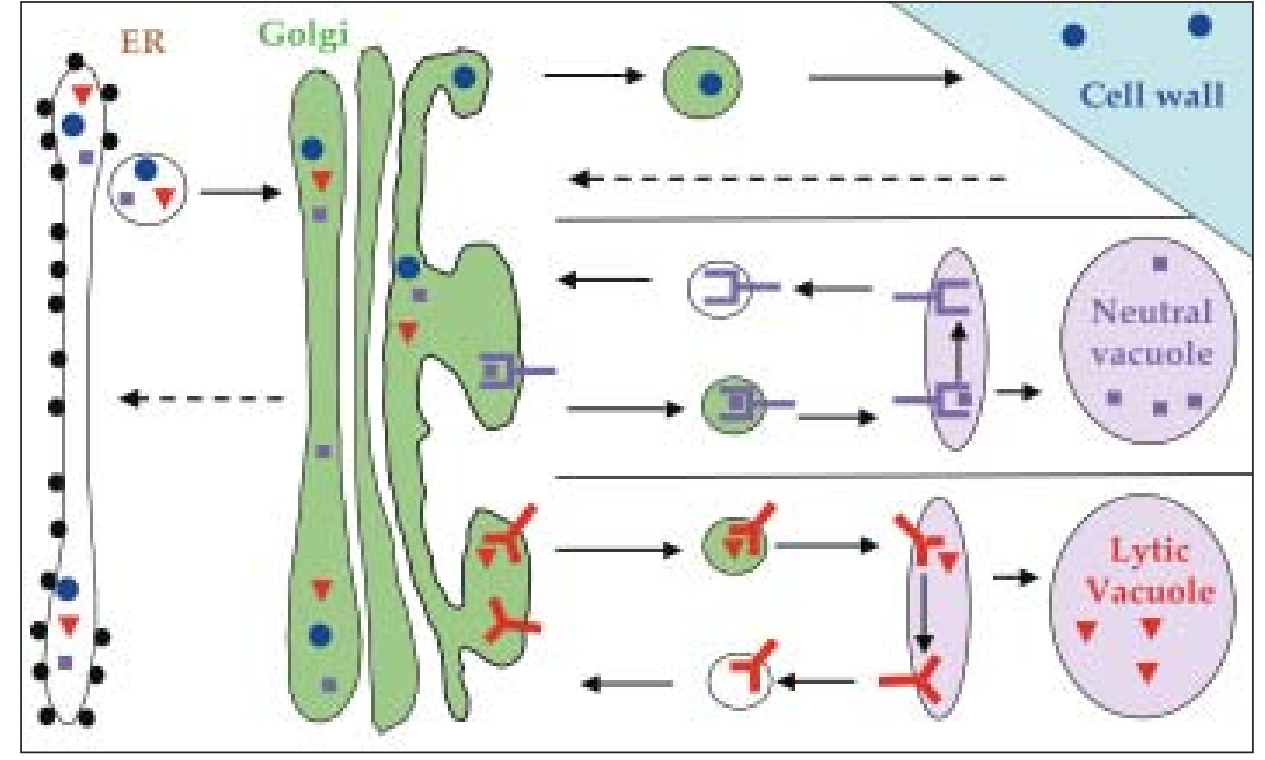

Fig. 3. Functional model of the secretory pathway and the pathways to two different vacuoles. Soluble secretory proteins are synthesized into the endoplasmatic reticulum (ER) by membranebound ribosomes, and transported to the Golgi apparatus by vesicles. In the Golgi, proteins are matured and sorted by various sorting systems. The default destination for soluble proteins without a specific sorting signal is the plasmalemma, leading to secretion into the cell wall (top). Proteins with a C-terminal vacuolar sorting signal (VSS) are sorted by a putative receptor into specific transport vesicles and transported to a pH-neutral vacuole, via a prevacuolar compartment (middle). The receptor is presumably recycled. Proteins with a sequence-specific VSS are sorted by a receptor of the VSR family into specific clathrin-coated vesicles and transported to an acidic prevacuolar compartment, where the receptor releases its ligand and recycles back to the Golgi (bottom). The vacuolar protein is further transported to the lytic vacuole. This last pathway corresponds to the pathways to lysosomes in animal cells and to the vacuole in yeast.

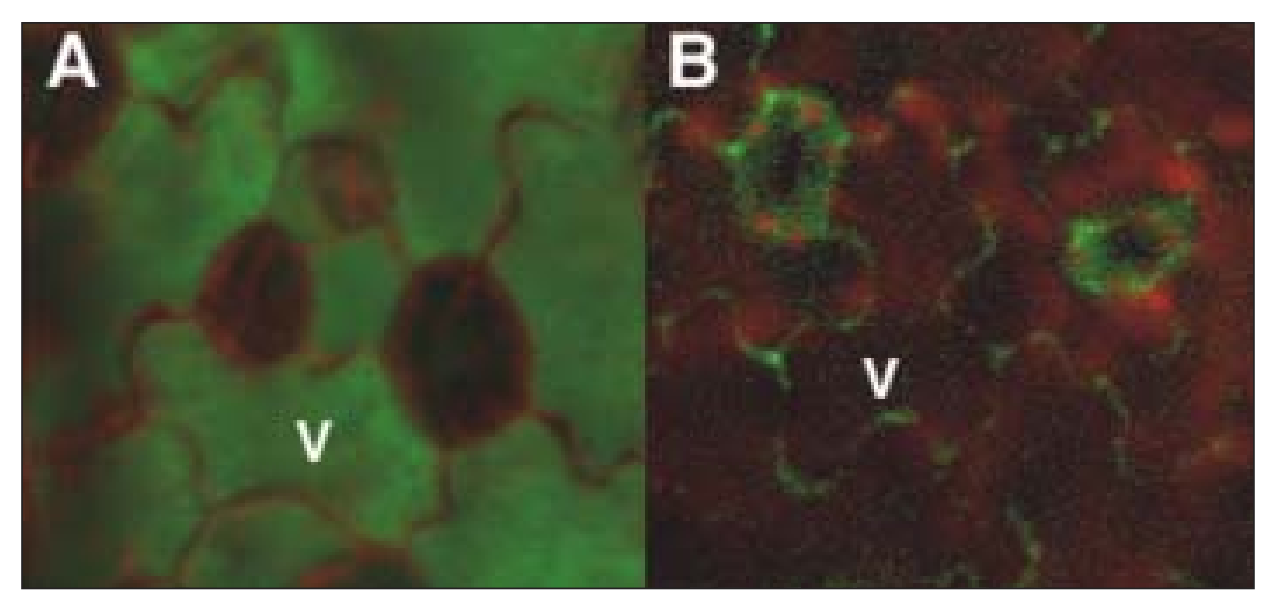

Fig. 4 Visualization of differential vacuolar targeting by the Green Fluorescent Protein (GFP). GFP fused to the sequence-specific vacuolar sorting signal (A, signal for the lytic vacuole) or to the $C$-terminal vacuolar sorting signal $(B$, signal for the neutral vacuole) were introduced into Arabidopsis thaliana. Leaves were visualized by scanning laser confocal microscopy (Leica DMR using the Leica TCS 4D operating system), using an FITC filter to visualize GFP and a TriTC filter to visualize chlorophyll. In the leaf epidermis, the large central vacuole (labeled $V$ in one cell of each part of the figure) of epidermal cells (jigsaw puzzle shaped cells) accumulates the marker for the lytic vacuole, while the marker for the neutral vacuole is confined to peripheral small compartments, but is visible in the central vacuole of guard cells (pairs of banana-shaped cells controlling the air flow into and out of the leaves). In contrast, in internal layers of the leaves (not shown), the central vacuole accumulates only the marker for neutral vacuoles. 
certain tissues of legumes or pumpkin is unclear, as these might actually be the vehicles of a third type of vacuolar sorting system.

\section{Functions of the Different Vacuoles}

Experiments in evacuolated protoplasts (plant cells isolated without cell walls) indicated that the regenerating vacuole is always of the acidic type, but also showed that after the end of cell expansion, the marker of the neutral vacuole, which had been confined to small peripheral vacuoles, now also could appear in the large central vacuole [27]. Similar observations were made in transgenic Arabidopsis plants: in expanding root or leaf hairs (trichomes), only the sequence-specific signal-GFP accumulated in the central vacuole, while again the other reporter accumulated in peripheral small vacuoles. After the end of expansion, the latter reporter also appeared in the central vacuoles [28]. These results suggest the possibility of either regulated fusion of preexisting different vacuoles or regulated convergence of the distinct transport pathways. The vacuolar system of plants has the potential of creating complex structures, such as storage vacuoles enclosing two different types of smaller vacuoles [31].

\section{Outlook}

The existence of different vacuole types and of corresponding specialized sorting systems is a distinctive feature of plants. We know little about the functions of the different vacuoles in different tissues and how plants modulate their use. The existence of small gene families of receptors suggest that the plants can also modulate the choice of proteins sent to a given vacuole. It is the specificity of the different vacuoles and their importance in different aspects of a plant's life that will keep us busy over the next years.

\section{Conclusion}

The laboratories of plant biochemistry and physiology of the University of Neuchâtel focus their main research projects on the biogenesis of two of the most plant-specific organelle systems. Other important research interests have not been discussed here, especially those associated with the National Center of Competence in Research (NCCR) 'Plant Survival', which are in part presented in the accompanying article by R. Tabacchi and co-workers [32].

\section{Acknowledgements}

Pictures for Fig. 4 were produced by Ricardo Flückiger. Our research is supported by grants from the Swiss National Science Foundation, FN-3100-065191.01 (J.-M.N.), a European Human Potential Network 'BioInteractions', grant OFES 01.0238 (J.-M.N.), Swiss National Science Foundation, FN-3100-067764.02 (F.K.) and ETH grant TH-23./01-3 (F.K.).

Received: July 17, 2003

[1] J. Bauer, A. Hiltbrunner, F. Kessler, Cell. Mol. Life. Sci. 2001, 58, 420.

[2] D.J. Schnell, D.N. Hebert, Cell 2003, 112, 833.

[3] A. Hiltbrunner, J. Bauer, M. AlvarezHuerta, F. Kessler, Biochem. Cell Biol. 2001, 79, 1.

[4] P. Jarvis, J. Soll, Biochim. Biophys. Acta 2002, 1590, 177.

[5] X. Chen, D.J. Schnell, Trends Cell Biol. 1999, 9, 222.

[6] D.J. Schnell, F. Kessler, G. Blobel, Science 1994, 266, 1007.

[7] F. Kessler, G. Blobel, Proc. Natl. Acad. Sci. USA 1996, 93, 7684

[8] D.T. Jackson, J.E. Froehlich, K. Keegstra, J. Biol. Chem. 1998, 273, 16583 .

[9] E. Nielsen, M. Akita, J. Davila-Aponte, K. Keegstra, EMBO J. 1997, 16, 935.

[10] L. Heins, A. Mehrle, R. Hemmler, R. Wagner, M. Kuchler, F. Hormann, D. Sveshnikov, J. Soll, EMBO J. 2002, 21, 2616.

[11] A. Caliebe, R. Grimm, G. Kaiser, J. Lübeck, J. Soll, L. Heins, EMBO J. 1997, 16,7342 .

[12] M. Kuchler, S. Decker, F. Hormann, J. Soll, L. Heins, EMBO J. 2002, 21, 6136.

[13] A. Kouranov, D.J. Schnell, J. Cell Biol. 1997, 139, 1677

[14] E. Schleiff, M. Jelic, J. Soll, Proc. Natl. Acad. Sci. USA 2003, 100, 4604.

[15] S.E. Perry, K. Keegstra, Plant Cell 1994, 6, 93.

[16] N. Sveshnikova, J. Soll, E. Schleiff, Proc. Natl. Acad. Sci. USA 2000, 97, 4973.

[17] Y.J. Sun, F. Forouhar, H. Li, S. Tu, Y.H. Yeh, S. Kao, H.L. Shr, C.C. Chou, C. Chen, C.D. Hsiao, Nat. Struct. Biol. 2002, 9, 95 .

[18] M. Smith, A. Hiltbrunner, F. Kessler, D.J. Schnell, J. Cell Biol. 2002, 159, 833.

[19] J. Bauer, A. Hiltbrunner, P. Weibel, P.-A. Vidi, M. Alvarez-Huerta, M.D. Smith, D.J. Schnell, F. Kessler, J. Cell Biol. 2002, 159,845 .

[20] A. Hiltbrunner, J. Bauer, P.-A. Vidi, S. Infanger, P. Weibel, M. Hohwy, F. Kessler, J. Cell Biol. 2001, 154, 309.

[21] F. Kessler, D.J. Schnell, Nat. Struct. Biol. 2002, $9,81$.

[22] P. Jarvis, L.J. Chen, H. Li, C.A. Peto, C. Fankhauser, J. Chory, Science 1998, 282, 100.

[23] J. Bauer, K. Chen, A. Hiltbrunner, E. Wehrli, M. Eugster, D. Schnell, F. Kessler, Nature 2000, 403, 203.

[24] J.-M. Neuhaus, E. Martinoia, in 'Encyclopedia of Life Sciences', Nature Publishing Group, London, 1999.
[25] G.P. Di Sansebastiano, N. Paris, S. MarcMartin, J.-M. Neuhaus, Plant J. 1998, 15, 449.

[26] K. Matsuoka, J.-M. Neuhaus, J. Exp. Bot. 1999, 50, 165.

[27] G.P. Di Sansebastiano, N. Paris, S. MarcMartin, J.-M. Neuhaus, Plant Physiol. 2001, 126, 78.

[28] R. Flückiger, M. De Caroli, G. Piro, G. Dalessandro, J.-M. Neuhaus, G.P. Di Sansebastiano, J. Exp. Bot. 2003, 54, 1577 .

[29] N. Paris, J.M. Neuhaus, Plant Mol. Biol. 2002, 50, 903.

[30] V. Laval, F. Masclaux, A. Serin, M. Carriere, C. Roldan, M. Devic, R.F. PontLezica, J.P. Galaud, J. Exp. Bot. 2003, 54 , 213.

[31] L. Jiang, T.E. Phillips, C.A. Hamm, Y.M. Drozdowicz, P.A. Rea, M. Maeshima, S.W. Rogers, J.C. Rogers, J. Cell Biol. 2001, 155, 991.

[32] S. Vogelsang, E. Abou-Mansour, P. Guerin, M.E. Hoballah, T. Turlings, R. Tabacchi, Chimia 2003, 57, 631. 